\title{
Correction to: Classical galactosemia: neuropsychological and psychosocial functioning beyond intellectual abilities
}

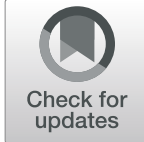

\author{
Mendy M. Welsink-Karssies ${ }^{1}$, Kim J. Oostrom², Merel E. Hermans ${ }^{3}$, Carla E. M. Hollak', Mirian C. H. Janssen ${ }^{5}$, \\ Janneke G. Langendonk ${ }^{6}$, Esmee Oussoren ${ }^{7}$, M. Estela Rubio Gozalbo ${ }^{8}$, Maaike de Vries ${ }^{9}$, Gert J. Geurtsen ${ }^{3}$ and \\ Annet M. Bosch ${ }^{1 *}$
}

\section{Correction to: Orphanet Journal of Rare Diseases (2020) 15:42 https://doi.org/10.1186/s13023-019-1277-0}

Following the original article's publication [1] the authors informed us of the following errors:

1. Author M. Estela Rubio Gozalbo's first and last names were captured incorrectly. The author's first names are M. Estela, while last names are Rubio Gozalbo.

The correct author's name has been updated in the original article [1] and shown in the author list of this Correction.

2. In Table 2 'Digit span' and 'GIT-2' are tests and should be preceded by a - just like the other tests in the table.

Furthermore, 'Responses' is not a domain and should therefore be deleted.

Finally, 'Cconceptual' should be corrected to 'conceptual'.

The table is shown here corrected.

3 . The grey box for 'test result better than expected' is missing from the legends of Tables 4 and 5 .

The two tables with their respective legends are shown here corrected.
4. In the 'Results' section under 'Demographics', the following sentence is incorrect: "In the pre-NBS group $(n=30)$ (diagnosis based on clinical symptoms), with the exception of the late diagnosed p.Ser135Leu patients, the diet was started at a median age of 10 days $[6,39]$." The references 6,39 are in fact a range. The sentence should therefore be corrected to read: "...the diet was started at a median age of 10 days (6-39)."

5. In Table S8 of the Additional file 1, some rows have shifted. 'Anxiety \& Depression' and 'Social Functioning' should be shown on separate lines. HADS should be in line with 'Anxiety \& Depression' and SRS with 'Social Functioning'.

In addition, subsequent references should be renumbered as $35-49$.

The corrected Additional file 1 accompanies this Correction.

The original article can be found online at https://doi.org/10.1186/s13023019-1277-0.

* Correspondence: a.m.bosch@amsterdamumcnl

'Department of Pediatrics, room H7-270, Amsterdam University Medical

Centre, MC, PO BOX 22660, 1100 DD Amsterdam, The Netherlands

Full list of author information is available at the end of the article

(c) The Author(s). 2020 Open Access This article is licensed under a Creative Commons Attribution 4.0 International License, which permits use, sharing, adaptation, distribution and reproduction in any medium or format, as long as you give appropriate credit to the original author(s) and the source, provide a link to the Creative Commons licence, and indicate if changes were made. The images or other third party material in this article are included in the article's Creative Commons licence, unless indicated otherwise in a credit line to the material. If material is not included in the article's Creative Commons licence and your intended use is not permitted by statutory regulation or exceeds the permitted use, you will need to obtain permission directly from the copyright holder. To view a copy of this licence, visit http://creativecommons.org/licenses/by/4.0/ The Creative Commons Public Domain Dedication waiver (http://creativecommons.org/publicdomain/zero/1.0/) applies to the data made available in this article, unless otherwise stated in a credit line to the data. 
Table 2 Cognitive Functioning Results

\begin{tabular}{|c|c|c|c|c|c|c|c|c|c|c|}
\hline Domain & $N$ & $\begin{array}{l}\text { Results } \\
\text { patients }\end{array}$ & $P$-value ${ }^{a}$ & $N$ & FSIQ 50-69 (Group 1) & $N$ & $\begin{array}{l}\text { FSIQ 70-85 } \\
\text { (Group 2) }\end{array}$ & N & $\begin{array}{l}\text { FSIQ > } 85 \\
\text { (Group 3) }\end{array}$ & $P$-value ${ }^{b}$ \\
\hline \multicolumn{11}{|l|}{ Learning \& Memory } \\
\hline - AVLT Immediate Recall & 19 & $46.0(9-61)$ & 0.029 & 4 & $40.0(9-46)$ & 11 & $45.00(35-61)$ & 4 & $49.50(47-51)$ & 0.121 \\
\hline - AVLT Delayed Recall & 19 & $46.0(15-65)$ & 0.545 & 4 & $49.5(15-58)$ & 11 & $46.00(34-65)$ & 4 & $50.00(43-55)$ & 0.956 \\
\hline - AVLT Delayed / Immediate & 19 & $52.0(38-65)$ & 0.445 & 4 & $55.5(43-62)$ & 11 & $52.00(38-65)$ & 4 & $52.00(39-62)$ & 0.803 \\
\hline - Digit span & 35 & $43.0(20-63)$ & $<0.0005^{c}$ & 8 & $30.00(20-57)$ & 15 & $43.00(27-60)$ & 12 & $48.50(33-63)$ & 0.017 \\
\hline \multicolumn{11}{|l|}{ Visuospatial functioning } \\
\hline - GIT-2 ${ }^{\mathrm{a}}$ spatial test & 19 & $36.0(23-50)$ & $<0.0005^{c}$ & 4 & $26.5(23-40)$ & 11 & $35.0(28-40)$ & 4 & $41.5(40-50)$ & 0.019 \\
\hline - Block design & 42 & $38.5(20-53)$ & $<0.0005^{c}$ & 9 & $30.0(20-40)$ & 17 & $37.0(27-50)$ & 16 & $37.0(33-53)$ & $<0.0005$ \\
\hline \multicolumn{11}{|l|}{ Executive functioning } \\
\hline \multicolumn{11}{|l|}{ Inhibition } \\
\hline - Stroop III (Inhibition) & 25 & $45.0(20-56)$ & $0.003^{c}$ & 6 & $27.0(20-49)$ & 13 & $48.00(22-56)$ & 6 & $46.0(35-53)$ & 0.078 \\
\hline - Stroop III/II (Interference) & 25 & $49.0(30-66)$ & 0.537 & 6 & $43.0(30-60)$ & 13 & $50.00(31-66)$ & 6 & $47.5(40-63)$ & 0.642 \\
\hline \multicolumn{11}{|l|}{ Cognitive flexibility } \\
\hline - WCST Total number of errors & 24 & $50.5(27-67)$ & 0.988 & 6 & $46.0(27-50)$ & 12 & $51.50(37-67)$ & 6 & $52.0(39-64)$ & 0.134 \\
\hline - WCST Perseverative responses & 24 & $51.0(30-81)$ & 0.626 & 6 & $46.0(33-52)$ & 12 & $53.00(30-81)$ & 6 & $52.0(35-73)$ & 0.278 \\
\hline $\begin{array}{l}\text { - WCST Percent conceptual level } \\
\text { responses }\end{array}$ & 24 & $49.5(27-64)$ & 0.951 & 6 & $48.0(27-51)$ & 12 & $52.00(39-64)$ & 6 & $51.0(37-62)$ & 0.270 \\
\hline - TMT B/A & 25 & $44.0(27-57)$ & $0.002^{c}$ & 6 & $45.0(27-50)$ & 13 & $43.00(27-57)$ & 6 & $48.5(40-57)$ & 0.510 \\
\hline - Letter fluency & 19 & $37.0(27-67)$ & $0.001^{\mathrm{c}}$ & 4 & $31.0(28-38)$ & 11 & $39.00(31-67)$ & 4 & $34.0(27-56)$ & 0.143 \\
\hline \multicolumn{11}{|l|}{ Mental Speed } \\
\hline - Stroop I (Color naming) & 25 & $40.0(25-61)$ & $0.001^{c}$ & 6 & $35.0(25-43)$ & 13 & $43.00(35-61)$ & 6 & $46.5(33-55)$ & 0.077 \\
\hline - Stroop II (Word reading) & 25 & $37.0(20-56)$ & $<0.0005^{c}$ & 6 & $30.0(20-40)$ & 13 & $43.00(20-56)$ & 6 & $39.0(33-56)$ & 0.063 \\
\hline - TMT A (Digit sequencing) & 25 & $52.0(20-67)$ & 0.352 & 6 & $50.5(20-59)$ & 13 & $56.00(43-67)$ & 6 & $47.5(33-67)$ & 0.173 \\
\hline - TMT B (Digit-Letter-Switching) & 25 & $45.0(20-58)$ & $0.003^{c}$ & 6 & $33.5(20-47)$ & 13 & $46.00(20-58)$ & 6 & $45.0(33-56)$ & 0.111 \\
\hline - Symbol search & 41 & $43.0(20-67)$ & $<0.0005^{c}$ & 9 & $23.0(20-50)$ & 17 & $40.00(27-67)$ & 15 & $47.0(40-60)$ & $0.001^{c}$ \\
\hline - Substitution & 42 & $40.0(23-57)$ & $<0.0005^{c}$ & 9 & $30.0(23-47)$ & 17 & $40.00(30-57)$ & 16 & $43.0(33-53)$ & 0.006 \\
\hline
\end{tabular}

Data reported in T-scores, median (ranges). ${ }^{\mathrm{a}}$ Patient data vs. normative data (T-score 50), ${ }^{\mathrm{b}}$ Comparison between FSIQ groups, ${ }^{\mathrm{C}}$ Significant after Bonferroni-Holm correction. FSIQ full scale IQ, AVLT auditory verbal learning test, GIT-Il groninger intelligentie test 2, Stroop stroop color word test, WCST wisconsin card sorting test, TMT trail making test 
Table 4 Individual Results, Adult Patients

\begin{tabular}{|c|c|c|c|c|c|c|c|c|c|c|c|c|c|c|c|c|c|c|c|}
\hline Patient $\rightarrow$ & 1 & 2 & 3 & 4 & 5 & $6^{2}$ & 7 & 8 & 9 & 10 & 11 & 12 & 13 & 14 & 15 & 16 & 17 & 18 & 19 \\
\hline VIQ & 103 & 101 & 72 & 100 & 77 & 90 & 86 & 72 & 83 & 64 & 68 & 81 & 81 & 70 & 66 & 86 & 83 & 93 & 81 \\
\hline PIQ & 104 & 77 & 72 & 85 & 60 & 72 & 75 & 62 & 75 & 58 & 60 & 75 & 87 & 70 & 68 & 75 & 77 & 77 & 81 \\
\hline \multicolumn{20}{|c|}{ Test results (corrected for FSIO): } \\
\hline \multirow{2}{*}{\multicolumn{20}{|c|}{$\begin{array}{l}\text { Executive functioning } \\
\text { Inhibition }\end{array}$}} \\
\hline & & & & & & & & & & & & & & & & & & \multicolumn{2}{|c|}{ Inhibition } \\
\hline \multirow{2}{*}{\multicolumn{20}{|c|}{$\frac{\text { Stroop III }}{\text { Stroop IIIIII }}$}} \\
\hline & & & & & & & & & & & & & & & & & & & \\
\hline \multicolumn{20}{|l|}{ Cognitive flexibility } \\
\hline - WCST Total Errors & & & & & & & & $\mathrm{x}$ & & & & & & & & & & & \\
\hline - WCST PR & & & & & & & & $\mathrm{X}$ & & & & & & & & & & & \\
\hline - WCST Percent CLR & & & & & & & & $\mathrm{x}$ & & & & & & & & & & & \\
\hline \multicolumn{20}{|l|}{- TMT B/A } \\
\hline - Letter fluency & & & & & & & & $\mathrm{x}$ & & & & & & & & & & & \\
\hline \multicolumn{20}{|l|}{ Learning \& Memory } \\
\hline - AVLT Immediate Recall & & & & & & & & $\mathrm{x}$ & & & & & & & & & & & \\
\hline - AVLT Delayed Recall & & & & & & & & $\mathrm{X}$ & & & & & & & & & & & \\
\hline - AVLT Delayed/Immediate & & & & & & & & $\mathrm{x}$ & & & & & & & & & & & \\
\hline \multirow{2}{*}{\multicolumn{20}{|c|}{$\frac{\text { Digit span }}{\text { Mental Speed }}$}} \\
\hline & & & & & & & & & & & & & & & & & & & \\
\hline \multicolumn{20}{|l|}{ - Stroop I } \\
\hline \multirow{2}{*}{\multicolumn{20}{|c|}{ - Stroop II }} \\
\hline & & & & & & & & & & & & & & & & & & & \\
\hline \multicolumn{18}{|l|}{$\frac{- \text { TMTA }}{- \text { TMT B }}$} & & \\
\hline \multicolumn{20}{|l|}{-Symbol search } \\
\hline \multirow{2}{*}{\multicolumn{20}{|c|}{$\begin{array}{l}\text {-Substitution } \\
\text { Visuospatial functioning }\end{array}$}} \\
\hline & & & & & & & & & & & & & & & & & & & \\
\hline \multicolumn{20}{|l|}{ GIT-2 spatial test } \\
\hline \multirow{2}{*}{\multicolumn{20}{|c|}{$\begin{array}{l}\text { Block design } \\
\text { Onestionnaire results func }\end{array}$}} \\
\hline & & & & & & & & & & & & & & & & & & & \\
\hline HADS* ${ }^{*}$ & & & & & & $\ldots$ & & $\mathrm{X}$ & & & & - & & & &.- & & -- & \\
\hline BRIEF-A* & - & & - & & - & - & & $\mathrm{x}$ & - & & & - & & & - & - & - & -- & - \\
\hline SRS-A* & & $\bar{x}$ & $\bar{x}$ & & $\bar{x}$ & - & & & & $\bar{x}$ & $\mathrm{X}$ & - & & $\bar{X}$ & & & - & - & \\
\hline
\end{tabular}

ap.Ser135Leu homozygous patient. VIQ Verbal IQ, PIQ Performal IQ, FSIQ Full Scale IQ, Stroop Stroop Color Word Test, WCST Wisconsin Card Sorting Test, PR Perseverative Responses, CLR Conceptual Level Responses, TMT Trail Making Test, AVLT Auditory Verbal Learning Test, GIT-II Groninger Intelligentie Test 2, HADS Hospital Anxiety and Depression Scale, BRIEF Behavior Rating Inventory of Executive Function, SRS Social Responsiveness Scale

$X$ no test result, $\square$ test result worse than expected, $\square$ test result better than expected, $\square$ test result as expected

*X no result, - - T-score on total scale in clinical range, - T-score on total scale in subclinical range, $\square \mathrm{T}$-score on total scale within normal range 
Table 5 Individual Results, Pediatric Patients

\begin{tabular}{|c|c|c|c|c|c|c|c|c|c|c|c|c|c|c|c|c|c|}
\hline Patient $\rightarrow$ & 20 & $21^{\mathrm{b}}$ & $22^{\mathrm{b}}$ & 23 & $24^{b}$ & 25 & 26 & 27 & 28 & 29 & 30 & 31 & 32 & 33 & 34 & $35^{\mathrm{a}}$ & 36 \\
\hline VIQ & 106 & \begin{tabular}{|l|}
80 \\
\end{tabular} & 96 & 55 & 85 & 98 & 81 & 60 & 90 & 102 & 106 & 92 & 74 & 72 & 81 & 70 & 95 \\
\hline PIQ & 93 & 100 & 85 & 85 & 97 & 85 & 59 & 75 & 77 & 89 & 99 & 86 & 61 & 85 & 95 & 57 & 98 \\
\hline \multicolumn{18}{|c|}{ Test results (corrected for FSIQ): } \\
\hline \multicolumn{18}{|l|}{ Executive functioning } \\
\hline \multicolumn{18}{|l|}{ Inhibition } \\
\hline - NI: Naming Total Errors & $\mathrm{X}$ & & & $\bar{X}$ & & $\bar{x}$ & & & & & $\bar{X}$ & $\bar{x}$ & $\mathrm{X}$ & $\mathrm{x}$ & $\bar{x}$ & $\mathrm{X}$ & $\mathrm{X}$ \\
\hline - NI: Inhibition Total Errors & $\mathrm{X}$ & & & $\mathrm{X}$ & & $\mathrm{X}$ & & & & & $\mathrm{X}$ & $\mathrm{X}$ & $\mathrm{X}$ & $\mathrm{X}$ & $\mathrm{X}$ & $\mathrm{X}$ & $\mathrm{X}$ \\
\hline - NI: Switching Total Errors & $\mathrm{X}$ & $\mathrm{X}$ & $\mathrm{X}$ & $\mathrm{X}$ & & $\mathrm{X}$ & & & & & $\mathrm{X}$ & $\mathrm{X}$ & $\mathrm{X}$ & $\mathrm{X}$ & $\mathrm{X}$ & $\mathrm{X}$ & $\mathrm{X}$ \\
\hline - Stroop III & $\mathrm{X}$ & $\mathrm{X}$ & $\mathrm{X}$ & $\mathrm{X}$ & $\mathrm{X}$ & $\mathrm{X}$ & $\mathrm{X}$ & $\mathrm{X}$ & $\mathrm{X}$ & $\mathrm{X}$ & $\mathrm{X}$ & & & & & & \\
\hline -Stroop III/II & $\mathrm{X}$ & $\bar{X}$ & $\bar{X}$ & $\bar{X}$ & $\mathrm{X}$ & $\bar{X}$ & $\bar{X}$ & $\bar{X}$ & $\bar{X}$ & $\bar{X}$ & $\bar{X}$ & & & & & & \\
\hline \multicolumn{18}{|l|}{ Cognitive flexibility } \\
\hline - NEPSY RS, Total Correct & $\mathrm{X}$ & $\mathrm{X}$ & $\mathrm{X}$ & $\mathrm{X}$ & & $\mathrm{X}$ & & & & & & $\mathrm{x}$ & $\mathrm{X}$ & $\mathrm{X}$ & $\mathrm{X}$ & $\mathrm{X}$ & $\mathrm{x}$ \\
\hline - WCST Total Errors & $\mathrm{X}$ & $\mathrm{X}$ & $\mathrm{X}$ & $\mathrm{X}$ & $\mathrm{X}$ & $\mathrm{X}$ & $\mathrm{X}$ & $\mathrm{X}$ & $\mathrm{X}$ & $\mathrm{X}$ & $\mathrm{X}$ & & & & & & \\
\hline - WCST PR & $\mathrm{X}$ & $\bar{X}$ & $\mathrm{X}$ & $\mathrm{X}$ & $\bar{X}$ & $\mathrm{X}$ & $\bar{x}$ & $\bar{x}$ & $\mathrm{X}$ & $\bar{X}$ & $\bar{X}$ & & & & & & \\
\hline -WCST Percent CLR & $\mathrm{X}$ & $\bar{X}$ & $\bar{X}$ & $\bar{X}$ & $\bar{X}$ & $\bar{X}$ & $\bar{X}$ & $\bar{x}$ & $\bar{X}$ & $\bar{X}$ & $\bar{X}$ & & & & & & \\
\hline- TMT B/A & $\bar{X}$ & $\bar{X}$ & $\bar{X}$ & $\bar{X}$ & $\bar{X}$ & $\bar{X}$ & $\bar{X}$ & $\bar{X}$ & $\bar{X}$ & $\bar{X}$ & $\bar{X}$ & & & & & & \\
\hline - Letter fluency & $\mathrm{X}$ & $\mathrm{X}$ & $\mathrm{X}$ & $\mathrm{X}$ & $\mathrm{X}$ & $\mathrm{X}$ & $\mathrm{X}$ & $\mathrm{X}$ & $\mathrm{X}$ & $\mathrm{X}$ & $\mathrm{X}$ & $\mathrm{X}$ & $\mathrm{X}$ & $\mathrm{X}$ & $\mathrm{X}$ & $\mathrm{X}$ & \\
\hline \multicolumn{18}{|l|}{ Attention } \\
\hline - NEPSY AA, Total Correct & $\mathrm{X}$ & & & $\bar{X}$ & & $\mathrm{X}$ & & & & & $\bar{X}$ & $\mathrm{X}$ & $\mathrm{X}$ & $\mathrm{X}$ & $\bar{X}$ & $\mathrm{X}$ & $\overline{\mathrm{X}}$ \\
\hline \multicolumn{18}{|l|}{ Learning \& Memory } \\
\hline - AVLT Immediate Recall & $\mathrm{X}$ & $\mathrm{X}$ & $\mathrm{X}$ & $\mathrm{X}$ & $\mathrm{X}$ & $\mathrm{X}$ & $\mathrm{X}$ & $\mathrm{X}$ & $\mathrm{X}$ & $\mathrm{X}$ & $\mathrm{X}$ & $\mathrm{X}$ & $\mathrm{X}$ & $\mathrm{X}$ & $\mathrm{X}$ & $\mathrm{X}$ & \\
\hline - AVLT Delayed Recall & $\mathrm{X}$ & $\mathrm{X}$ & $\mathrm{X}$ & $\mathrm{X}$ & $\mathrm{X}$ & $\mathrm{X}$ & $\mathrm{X}$ & $\mathrm{X}$ & $\mathrm{X}$ & $\mathrm{X}$ & $\mathrm{X}$ & $\mathrm{x}$ & $\mathrm{X}$ & $\mathrm{X}$ & $X$ & $\mathrm{X}$ & \\
\hline - AVLT Delayed/Immediate & $\mathrm{X}$ & $\bar{X}$ & $\mathrm{X}$ & $\bar{X}$ & $\bar{x}$ & $\mathrm{X}$ & $\bar{x}$ & $\bar{x}$ & $\mathrm{X}$ & $\mathrm{X}$ & $\bar{X}$ & $\mathrm{X}$ & $\bar{X}$ & $\bar{X}$ & $\bar{x}$ & $\mathrm{X}$ & \\
\hline - Digit span & $\mathrm{X}$ & $\bar{X}$ & & $\bar{X}$ & $\bar{X}$ & & & & & & & & & & & & \\
\hline \multicolumn{18}{|l|}{ Mental Speed } \\
\hline - NI: Naming TCT & $\mathrm{X}$ & & & $\mathrm{X}$ & & $\mathrm{X}$ & & & & & $\mathrm{X}$ & $\mathrm{X}$ & $\mathrm{X}$ & $\mathrm{X}$ & $\mathrm{X}$ & $\mathrm{X}$ & $\mathrm{x}$ \\
\hline- NI: Inhibition TCT & $\mathrm{X}$ & & & $\bar{X}$ & & $\bar{X}$ & & & & & $\bar{X}$ & $\mathrm{X}$ & $\bar{X}$ & $\bar{X}$ & $\bar{X}$ & $\mathrm{X}$ & $\bar{x}$ \\
\hline -NI: Switching TCT & $\mathrm{X}$ & $\mathrm{X}$ & $\mathrm{X}$ & $\mathrm{X}$ & & $\mathrm{X}$ & & & & & $\mathrm{X}$ & $\mathrm{X}$ & $\mathrm{X}$ & $\mathrm{X}$ & $\mathrm{X}$ & $\mathrm{X}$ & $\mathrm{X}$ \\
\hline Stroop I & $\mathrm{X}$ & $\mathrm{X}$ & $\mathrm{X}$ & $\mathrm{X}$ & $\bar{X}$ & $\bar{X}$ & $\bar{X}$ & $\bar{x}$ & $\mathrm{X}$ & $\mathrm{X}$ & $\mathrm{X}$ & & & & & & \\
\hline Stroop II & $\mathrm{X}$ & $\mathrm{X}$ & $\mathrm{X}$ & $\mathrm{X}$ & $\mathrm{x}$ & $\mathrm{x}$ & $x$ & $\mathrm{X}$ & $\mathrm{X}$ & $\mathrm{X}$ & $\mathrm{X}$ & & & & & & \\
\hline -TMT A & $\mathrm{X}$ & $\mathrm{X}$ & $\mathrm{X}$ & $\mathrm{X}$ & $\mathrm{X}$ & $\mathrm{X}$ & $\mathrm{X}$ & $\mathrm{X}$ & $\mathrm{X}$ & $\mathrm{X}$ & $\mathrm{X}$ & & & & & & \\
\hline- TMT B & $\mathrm{X}$ & $\bar{X}$ & $\bar{X}$ & $\bar{X}$ & $\bar{X}$ & $\bar{X}$ & $\bar{X}$ & $\bar{X}$ & $\mathrm{X}$ & $\mathrm{X}$ & $\bar{X}$ & & & & & & \\
\hline \multicolumn{18}{|l|}{ - Symbol search } \\
\hline \multirow{2}{*}{\multicolumn{18}{|c|}{$\begin{array}{l}\text {-Substitution } \\
\text { Visuospatial functioning }\end{array}$}} \\
\hline & & & & & & & & & & & & & & & & & \\
\hline - GIT-2 spatial test & $\mathrm{X}$ & $\mathrm{X}$ & $\mathrm{X}$ & $\mathrm{X}$ & $\mathrm{X}$ & $\mathrm{X}$ & $\mathrm{X}$ & $\mathrm{X}$ & $\mathrm{X}$ & $\mathrm{X}$ & $\mathrm{X}$ & $\mathrm{X}$ & $\mathrm{X}$ & $\mathrm{X}$ & $\mathrm{X}$ & $\mathrm{X}$ & \\
\hline \multirow{2}{*}{\multicolumn{18}{|c|}{\begin{tabular}{l|l|l} 
- Block design & & \\
Olestionale &
\end{tabular}}} \\
\hline \multicolumn{17}{|c|}{ Questionnaire results (uncorrected for FSIQ): } & \\
\hline CBCL $6=18 y^{3}$ & $\mathrm{X}$ & & & 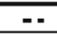 & $\mathrm{X}$ & $\bar{x}$ & & $\bar{X}$ & & & & & $\overline{--}+$ & 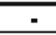 & & & \\
\hline YSR* & $\mathrm{X}$ & $\mathrm{X}$ & $\mathrm{X}$ & $\mathrm{X}$ & $\mathrm{x}$ & $\mathrm{X}$ & $\mathrm{X}$ & $\mathrm{x}$ & $\mathrm{X}$ & $\mathrm{X}$ & $\mathrm{X}$ & $\mathrm{X}$ & $\mathrm{x}$ & & & & $\mathrm{x}$ \\
\hline BRIEF-P* & - & & & $\mathrm{X}$ & $\mathrm{X}$ & $\mathrm{X}$ & $\mathrm{X}$ & $\mathrm{X}$ & & & & $\mathrm{X}$ & $\mathrm{X}$ & $\mathrm{X}$ & $\mathrm{X}$ & $\mathrm{X}$ & $\mathrm{X}$ \\
\hline BRIEF $^{*}$ & $\mathrm{X}$ & $\mathrm{X}$ & $\mathrm{X}$ & $\mathrm{X}$ & $\bar{x}$ & $\mathrm{X}$ & $\bar{x}$ & $\bar{x}$ & $\mathrm{X}$ & $\bar{X}$ & $\mathrm{X}$ & $\mathrm{X}$ & $\mathrm{X}$ & & & & - \\
\hline SRS-2* & & $\bar{X}$ & & $\mathrm{X}$ & & & & - & & - & & & & & & & \\
\hline
\end{tabular}

${ }^{a}$ p.Ser135Leu homozygous patient, ${ }^{\text {b }}$ Variant patient, VIQ Verbal IQ, PIQ Performal IQ, FSIQ Full Scale IQ, NI NEPSY Inhibition, Stroop Stroop Color Word Test, RS Response Set, AA Auditory Attention, WCST Wisconsin Card Sorting Test, PR Perseverative responses, CLR Conceptual Level Responses, TMT Trail Making Test, AVLT Auditory Verbal Learning Test, TCT Total Completion Time, GIT-II Groninger Intelligentie Test 2, CBCL 6-18y Child Behavior Checklist 6-18 years, YSR Youth Self Report, BRIEF Behavior Rating Inventory of Executive Function, SRS Social Responsiveness Scale

$\mathrm{X}$ no test result, $\square$ test result worse than expected, $\square$ test result better than expected, $\square$ test result as expected

${ }^{*} \mathrm{X}$ no result, - - T-score on total scale in clinical range, - T-score on total scale in subclinical range, $\square \mathrm{T}$-score on total scale within normal range

\section{Supplementary information}

Supplementary information accompanies this paper at https://doi.org/10. 1186/s13023-020-01447-z.

Additional file 1: Table S8. The Neuropsychological Assessment.

\section{Author details}

'Department of Pediatrics, room H7-270, Amsterdam University Medical Centre, MC, PO BOX 22660, 1100 DD Amsterdam, The Netherlands.

${ }^{2}$ Psychosocial Department, Emma Children's Hospital, Amsterdam UMC, University of Amsterdam, Amsterdam, The Netherlands. ${ }^{3}$ Department of Medical Psychology, Amsterdam UMC, University of Amsterdam, Amsterdam, The Netherlands. ${ }^{4}$ Department of Internal Medicine, Division of Endocrinology and Metabolism, Amsterdam UMC, University of Amsterdam, Amsterdam, The Netherlands. ${ }^{5}$ Department of Internal Medicine, Radboud University Medical Center, Nijmegen, The Netherlands. ${ }^{6}$ Department of Internal Medicine, Center for Lysosomal and Metabolic Diseases, Erasmus MC, University Medical Center Rotterdam, Rotterdam, The Netherlands.

'Department of Pediatrics, Center for Lysosomal and Metabolic Diseases,
Erasmus MC, University Medical Center Rotterdam, Rotterdam, The Netherlands. ${ }^{8}$ Department of Pediatrics and Department of Clinical Genetics, Maastricht University Medical Center, Maastricht, The Netherlands. ${ }^{9}$ Department of Pediatrics, Radboud University Medical Center, Nijmegen, The Netherlands.

Published online: 07 September 2020

Reference

1. Welsink-Karssies MM, et al. Classical galactosemia: neuropsychological and psychosocial functioning beyond intellectual abilities. Orphanet J Rare Dis. 2020;15:42 https://doi.org/10.1186/s13023-019-1277-0. 